\title{
Ultraviolet and optical observations of tidal disruption events
}

\author{
S. Gezaria \\ Department of Astronomy, University of Maryland, College Park, MD 20742-2421, USA
}

\begin{abstract}
Tidal disruption events are expected to produce a luminous flare of radiation from fallback accretion of tidally disrupted stellar debris onto the central supermassive black hole. The first convincing candidates for tidal disruption events were discovered in the soft X-rays: large-amplitude, luminous, extremely-soft X-ray flares from inactive galaxies in the ROSAT All-Sky survey. However, the sparsely sampled light curves and lack of multiwavelength observations for these candidates make it difficult to directly constrain the parameters of their events (e.g., Eddington ratio, mass of the black hole, type of star disrupted). Here I present a review of the recent progress made in studying tidal disruption events in detail from taking advantage of widefield, multi-epoch observations of UV and optical surveys (GALEX, SDSS, PTF, Pan-STARRS1) to measure well-sampled light curves, trigger prompt multiwavelength follow-up observations, and measure rates. I conclude with the promising potential of the next generation of optical synoptic surveys, such as LSST, to probe black hole demographics with samples of thousands of tidal disruption events.
\end{abstract}

\section{INTRODUCTION}

The characteristic temperature of a disk of stellar debris accreting onto a supermassive black hole is $T_{\text {eff }} \approx$ $2.5 \times 10^{5} M_{6}^{1 / 12} r_{\star}^{-1 / 2} m_{\star}^{-1 / 6}\left(r / r_{\mathrm{T}}\right)^{-1 / 2} \mathrm{~K}$, where $M_{6}=$ $M_{\mathrm{BH}} /\left(10^{6} M_{\odot}\right), r_{\star}=R_{\star} / R_{\odot}, m_{\star}=M_{\star} / M_{\odot}$, and $r / r_{\mathrm{T}}$ is the ratio of the radius of the disk to the tidal disruption radius, $r_{\mathrm{T}} \sim R_{\star}\left(M_{\mathrm{BH}} / M_{\star}\right)^{1 / 3}$. Thermal emission with this temperature peaks in the soft $\mathrm{X}$-rays, and is thus the ideal waveband to search for such events. Indeed, the first candidates for tidal disruption events (TDEs) were discovered in the 0.1-2.4 keV band by the ROSAT AllSky Survey. ROSAT detected luminous X-ray outbursts from several apparently inactive galaxies whose extremely soft spectra, dramatic fading on the timescale of years, and rate of $\approx 10^{-4}$ per year per galaxy were consistent with the theoretical expectations for TDEs. Several more candidates have been subsequently discovered with the XMM-Newton Slew Survey and in searches of archival Chandra data (see review talk by S. Komossa).

However, even the best of these candidates have sparsely light curves, with a typical time sampling of years (see Figure 1). One of the most exciting aspects of TDEs is the potential to use them as signposts for dormant massive black holes lurking otherwise undetectable in the centers of galaxies. Numerical simulations have shown that the mass accretion rate of stellar debris onto the black hole is well determined by only a few parameters: $M_{\mathrm{BH}}, M_{\star}, R_{\star}, \gamma, \beta$, where $\gamma$ is the polytropic exponent of the star, and $\beta=$ $r_{\mathrm{T}} / r_{\mathrm{p}}$, and $r_{\mathrm{p}}$ is the pericenter of the star's orbit. For stars on the main sequence, the relationship between $\gamma$ and $M_{\star}$, along with the mass-radius relation, causes a degeneracy, which along with a weak dependence on $\beta$, results in a mass accretion rate $(\dot{M})$ determined almost entirely by $M_{\mathrm{BH}}$ (Guillochon \& Ramirez-Ruiz 2012). Thus model fits to the flare light curve can in principle be used to weigh the central black hole. This is particularly useful since in the

a e-mail: suv@astro.umd.edu mass range where a black hole can disrupt a star before it passes the event horizon, $M_{\mathrm{BH}}<10^{8} M_{\odot}$, the $M_{\mathrm{BH}}-\sigma_{\star}$ relation from local galaxy dynamical measurements of $M_{\mathrm{BH}}$ is poorly constrained.

In practice, however, there are additional uncertainties on how $\dot{M}$ translates to a bolotmetric luminosity $\left(L_{\mathrm{bol}}\right)$ and finally into luminosity density $\left(L_{v}\right)$ in a particular bandpass, and how this depends on the mode of accretion (i.e. sub-Eddington or super-Eddington) (Strubbe \& Quataert 2009; Lodato et al. 2011). The power-law index, $n$, of the $t^{-n}$ decline of the light curve will vary from $n=5 / 3$ for $L_{\text {bol }}$ and $n=5 / 12$ for $L_{\mathrm{opt}}$ for sub-Eddington fallback, to $n=5 / 9$ for $L_{\text {bol }}$ and $n=35 / 36$ for $L_{\text {opt }}$ for a super-Eddington outflow. However, we can use TDEs as a cosmic laboratory to study accretion physics, the formation of accretion disks, and in some cases, the connection between accretion onto a black hole and jet formation. In order to do this, we require much more detailed light curves, and multiwavelength follow-up observations. For example, follow-up spectroscopic observations can detect signatures of photoionized unbound debris and circumnuclear material. Our strategy for tackling this problem has been to "look underneath the lamppost", and take advantage of existing wide-field multi-epoch UV and optical survey data to search for events and characterize them in more detail.

\section{SEARCHING UNDER THE LAMPPOST}

\subsection{UV/Optical Candidates}

Our first effort to search for TDEs at longer wavelengths was with the GALEX Deep Imaging Survey. The wide-field of view (1.2 deg), excellent sensitivity, and multi-epoch observing cadence enabled the discovery of extragalactic transients in the UV for the first time. We also took advantage of the synergy between GALEX DIS and the CFHT Supernova Legacy Survey (SNLS) 


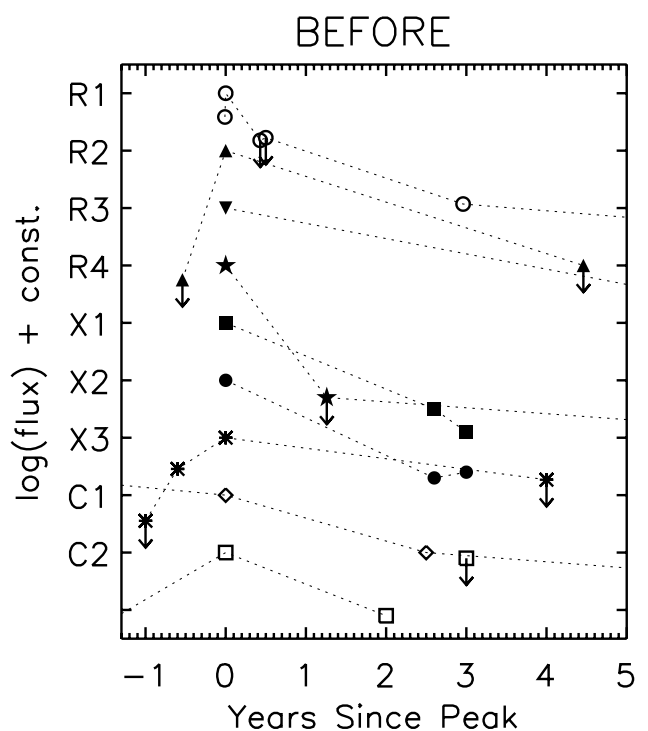

Figure 1. Sparsely sampled light curves of tidal disruption event candidates discovered in X-ray (ROSAT, XMM-Newton, Chandra) surveys.

in the optical. These surveys had $3 \mathrm{deg}^{2}$ of spatial and temporal overlap, such that we could extract simultanous optical light curves for any UV flaring candidates. By comparing yearly epochs of GALEX DIS images, we detected luminous flares from 3 galaxies which showed no other signs of activity from their nucleus. For 2 of the flares we could extract their densely sampled optical light curves from SNLS, cadence of 5 days, and found that they were well described by a power-law decline with time. The exact power-law index $n$ was less well constrained due to the loose constraints on the rise-time of the flares. Chandra Xray follow-up observations of these candidates measured upper limits on their hard X-ray emission that put further constraints on the presence of an AGN, and were consistent with a $<5 \times 10^{5} \mathrm{~K}$ thermal blackbody spectrum (Gezari et al. 2006, 2008, 2009).

In van Velzen et al. (2011) we present a search of archival SDSS Stripe 82 multi-epoch imaging over $300 \mathrm{deg}^{2}$. Note that when searching in the optical waveband one must contend with contrasting against the bright host galaxy light, the fact that the optical waveband is further down the Rayleigh-Jeans tail where the flares are intrinsically fainter, and there is a larger source of contamination from supernovae $(\mathrm{SNe})$. In this search, two flares were detected from galaxy nuclei with no other evidence of AGN activity. The light curves were remarkably similar to the GALEX-selected events, and we demonstrated that their variability statistics, spatial separation from the host, colors, and color-evolution could be used to select a parameter space that distinguished them from more common interloping $\mathrm{SNe}$ and AGNs. This is especially important for reducing the contamination rate to a maneagable number for targeted follow-up observations for classification purposes.

The Palomar Transient Factory (PTF) has been monitoring $2700 \mathrm{deg}^{2}$ of sky with a 5-day cadence in the $r$-band, and spectroscopically following up transients of interest (see talk by S.B. Cenko). A luminous optical outburst was detected from the nucleus of a star-forming galaxy with an SED fitted by a blackbody with $T_{\mathrm{BB}}>$ $2 \times 10^{4} \mathrm{~K}$ (Cenko et al. 2012). In contrast with the previous UV/optical and X-ray candidates discussed above, however, it was also detected as a luminous hard $\mathrm{X}$-ray transient in follow-up observations with Swift, with a power-law spectrum with $\Gamma=1.8$ similar to an AGN spectrum. In addition, the light curve in this event is also distinct, with the flare duration in the optical of less than 10 days. The properties of this event deviate from the expectations from the long-lived fallback accretion phase in a TDE, and may be related to a more brief "superEddington" outflow phase discussed in L. Strubbe's talk.

Motivated by our serendipitous discoveries with GALEX DIS and CFHT SNLS, we designed a dedicated GALEX Time Domain Survey (TDS) in the $N U V$ in spatial and temporal overlap with the Pan-STARRS1 Medium Deep Survey (MDS) in the $g, r, i, z$, and $y$ bands. GALEX TDS and Pan-STARRS1 MDS are well-matched in survey area, depth, and cadence (see Table 1). GALEX monitored 6 out of the 10 Pan-STARRS1 Medium Deep Fields, each with a field of view of $7 \mathrm{deg}^{2}$, with a cadence of 2 days during the windows of visibility. This coordinated wide-field monitoring has yielded the early detection of a Type IIP SN (Gezari et al. 2010), as well as a spectacular TDE candidate PS1-10jh reported in Gezari et al. (2012).

PS1-10jh was detected by GALEX as an $N U V$ transient with an amplitude of $>6.4 \mathrm{mag}$. This amplitude is an extreme outlier compared to the variability behavior of the AGNs and quasars in the GALEX TDS sample. The PS1 MDS first detected the flare coincident with the nucleus of a galaxy on 2010 May 31, and followed its rise to the peak on 2010 July 12, and its subsequent decay for over a year later. The flare was distinct from $\mathrm{SNe}$ (which cool from expansion of their ejecta) in the relatively constant blue colors of the flare, including a nearly constant $N U V-r$ color, indicating that the flare remained hotter than 30,000 K throughout the event. We triggered Director's Discretionary Time Chandra observations of the event which detected no hard X-ray emission, a factor of 20 below what would be expected for an AGN with a similar UV luminosity. We were able to identify the nature of the host galaxy from multi-epoch spectroscopy with the MMT telescope. As the hot flare faded, the host galaxy was revealed to be an early-type galaxy at $z=0.1696$. This also indicated that the broad emission line features seen to fade with the hot flare were in fact He II $\lambda 4686,3203$ emission lines. This indicated that in fact the fast moving gas photoionized by the flare was composed of mostly helium, with a hydrogen mass fraction of $<0.2$. This suggested that we were seeing photoionized debris from the tidal disruption of the helium-rich stellar core of a star that had lost its hydrogen envelope. Given the intermediate age of the host galaxy stellar population, and the lack of evidence for current star formation, the star was not likely a massive star that had lost its envelope through winds, but was likely a star that had lost its envelope through a previous tidal interaction with the central SMBH.

The excellent quality of the flare light curve together with information about the type of star disrupted enabled 
Table 1.

\begin{tabular}{|c|c|c|c|c|c|c|}
\hline Survey & $\begin{array}{l}\text { Field of View } \\
\text { (deg) }\end{array}$ & $\begin{array}{c}\text { plate scale } \\
(\operatorname{arcsec} / \text { pixel })\end{array}$ & $\begin{array}{l}\text { PSF FWHM } \\
\text { (arcsec) }\end{array}$ & $\begin{array}{c}m_{\text {lim }} \text { per Epoch } \\
\text { (mag) }\end{array}$ & $\begin{array}{c}\text { Cadence } \\
\text { (days) }\end{array}$ & $\begin{array}{r}\text { Seasonal Visibility } \\
\text { (months) }\end{array}$ \\
\hline GALEX TDS & 1.1 & 1.5 & 5.3 & 23.3 & 2 & $\sim 1$ \\
\hline PS1 MDS & 3.3 & 0.258 & 1.0 & 23.0 & 3 & $\sim 6$ \\
\hline
\end{tabular}

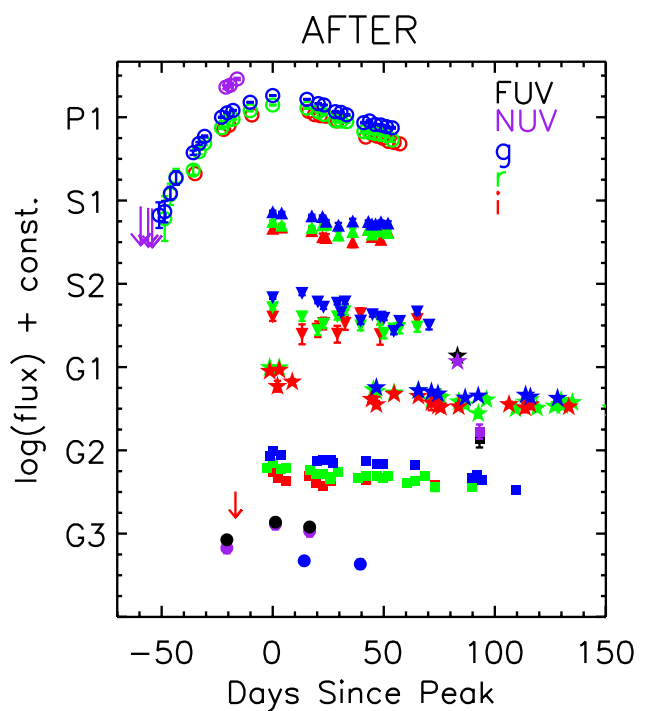

Figure 2. Densely sampled light curves of tidal disruption event candidates discovered in ultraviolet (GALEX), and optical (SDSS, PS1) surveys.

us to model the flare with the mass accretion rate calculated from numerical simulations for the tidal disruption of a stripped stellar core around a $2.8 \times 10^{6} M_{\odot}$ black hole. This also indicated that the peak luminosity of the event was close to Eddington, and the total material accreted was greater than $6 \%$ of the star disrupted. This is the first demonstration of the capability to use TDEs to in fact weigh their central black holes that would be otherwise hidden from view. In Figure 3 we show the black hole mass derived for different models in comparison to the large range of black hole mass allowed by the $M_{\mathrm{BH}}-M_{\text {bulge }}$ relation of $(2-8) \times 10^{6} M_{\odot}$.

\subsection{Uniform Properties}

Given that the candidates described above were selected via different surveys, at different wavebands, and different cadences, it is surprising how uniform their optical light curves are. Figure 2 shows the UV and optical light curves of the 6 candidates from GALEX, SDSS, and PanSTARRS1, all of which show constant blue colors and power-law declining light curves. In only one case, PS110jh (P1 in Figure 2), do we catch the rising portion of the light curve, which was critical for constraining the model fits, which in turn allow us to constrain the central black hole mass. The rising portion of the light curve is also necessary to constrain the power-law index of the decline, which is diagnostic of the mode of the accretion. In addition the UV/optical spectral energy distributions (SEDs) of these candidates are similar in that they can be fitted with the Rayleigh-Jeans tail of blackbody emission of $T_{\mathrm{BB}}>(2-5) \times 10^{4} \mathrm{~K}$. Note that these are lower

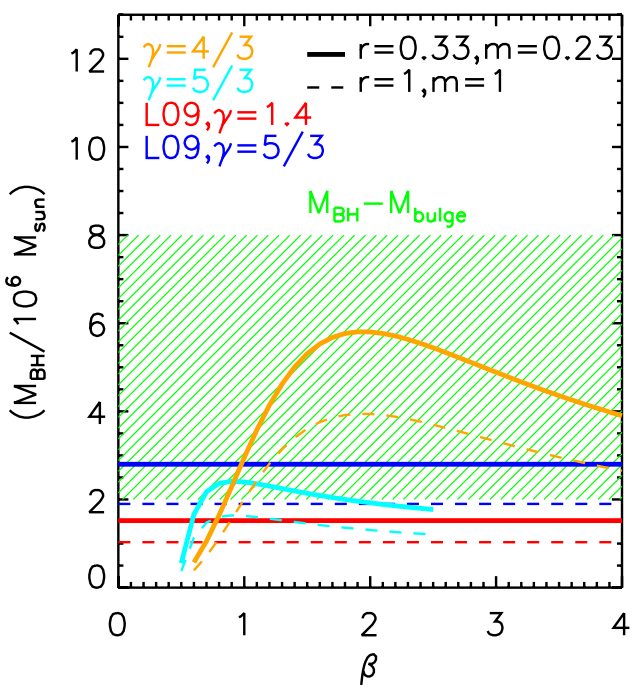

Figure 3. Mass of central black hole in PS1-10jh as derived from fits of the flare light curve to different models for $\dot{M}$. Hatched region shows the black hole mass range determined from the locally-established $M_{\mathrm{BH}}-M_{\text {bulge }}$ relation.

limits, since the temperature is not well constrained on the Rayleigh-Jeans tail, and is also very sensitive to internal extinction in the system. Even so, the X-ray upper limits in these systems constrain them to $T_{\mathrm{BB}}<5 \times 10^{5} \mathrm{~K}$.

\section{CANDIDATE DEMOGRAPHICS}

In Figure 4, we show a summary of the demographics of all of the candidates discovered so far by various means, from the optical to the hard X-rays. We plot bolometric luminosity versus peak temperature. In the case of the "relativistic" Swift candidate J2058.4 + 0516 (see talk by A. Levan), we plot the thermal component of the outburst detected in the optical band. The dashed black lines show the Eddington luminosity for a $10^{6}, 10^{7}$, and $10^{8} M_{\odot}$ black hole, respectively. The hard X-ray luminosity of the Swift candidate shown in the purple hatched region is clearly super-Eddington, and is likely beamed emission. While the luminosities of the X-ray and UV/optical candidates are similar, in the range of $10^{43-44} \mathrm{ergs} \mathrm{s}^{-1}$, their characteristic temperatures are quite different, with the X-ray-selected candidates having temperatures a factor of 10 higher. If the candidates had a characteristic radius, then they should follow the cyan diagonal lines, which show the luminositytemperature relation at the tidal disruption radius for a $10^{6}, 10^{7}$, and $10^{8} M_{\odot}$ black hole. Red dashed lines show the luminosity-temperature relation at the Schwartzschild radius $\left(r_{\mathrm{S}}\right)$ for a $10^{6}, 10^{7}$, and $10^{8} M_{\odot}$ black hole. Note that for a $10^{8} M_{\odot}$ black hole $r_{\mathrm{T}}=r_{\mathrm{S}}$.

There appears to be an issue with both the UV/optical and X-ray selected populations of TDE canddidates. The UV/optically selected candidates appear to have characteristic radii $>10 R_{\mathrm{T}}$, while the X-ray selected 


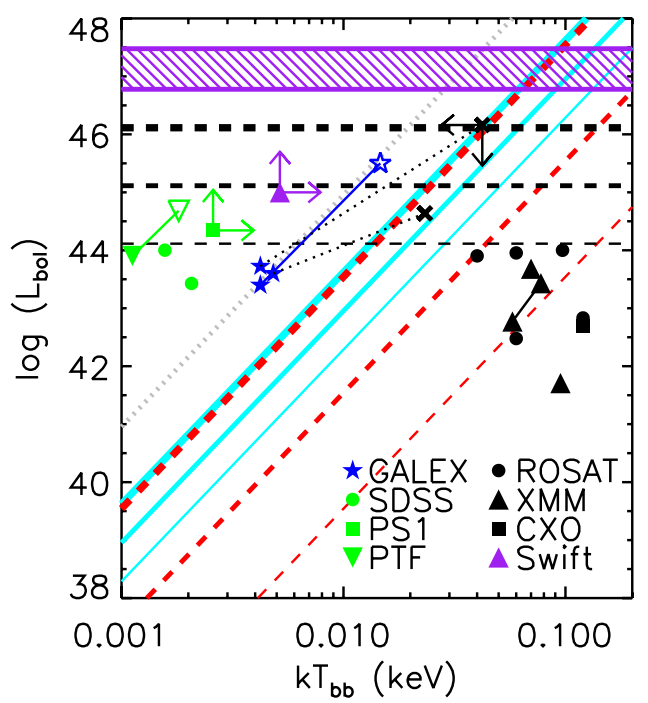

Figure 4. Demographics of tidal disruption event candidates discovered in X-ray (ROSAT, XMM-Newton, Chandra), ultraviolet (GALEX), and optical (SDSS, PTF, PS1) surveys.

candidates appear to have radii $<R_{\mathrm{S}}$. This may indicate that their effective temperatures do not correspond to a simple blackbody SED. The bimodal characteristic temperature distribution for the TDE candidates is best explained as a selection bias. However, the important question that must be now be addressed is if this represents two different populations, or different components of the same population. Prompt multiwavelength follow-up (or parallel wide-field monitoring) of future UV/optical and X-ray selected candidates is critical for answering this question.

\section{FUTURE SURVEYS}

The future is bright for detecting large samples of TDEs. The Large Synoptic Suervey Telescope (LSST) will have a similar depth and cadence as PS1 MDS, but with over 250 times the area. Van Velzen et al. (2011) scale the detection rate in SDSS Stripe 82 to LSST to determine a detection rate up to thousands of TDEs per year! However, this also translates to the detection of millions of $\mathrm{SNe}$ and quasars. Thus filtering and automated classification will be key tools for allocating limited follow-up resources. Futhermore, parallel spacebased wide-field monitoring in the soft X-rays and UV would be powerful for discriminating TDE candidates from peculiar $\mathrm{SNe}$ coincident with the nuclei of galaxies, the major source of contamination, and for searching for jetted TDE events. We have demonstrated with PS1 that detailed optical light-curves, that follow the rise and fall of a TDE flare with a cadence of days, can in fact yield modeldependent black hole mass measurements with smaller errors than indirect scaling relations such as the locallyestablished $M_{\mathrm{BH}}-M_{\text {bulge }}$ relation. Furthermore, a large numbers of TDE detections will enable us to probe the tails of the black-hole mass distribution, from intermediatemass black holes in the centers of dwarf galaxies, to supermassive spinning black holes.

\section{References}

[1] B. Cenko et al., MNRAS 420, (2012) 2684

[2] S. Gezari et al., Nature 485, (2012) 217

[3] S. Gezari et al., ApJ 720, (2010) L77

[4] S. Gezari et al., ApJ 698, (2009) 1367

[5] S. Gezari et al., ApJ 676, (2008) 944

[6] S. Gezari et al., ApJ 653, (2006) L25

[7] J. Guillochon \& E. Ramirez-Ruiz, ApJ, (2012) submitted (arXiv:1206:2350)

[8] G. Lodato \& E.M. Rossi, MNRAS 410, (2011) 359

[9] L. Strubbe \& E. Quataert, MNRAS 400, (2009) 207

[10] S. van Velzen et al., ApJ 741, (2011) 73 